\title{
Téoros
}

Revue de recherche en tourisme

\section{L’hébergement socio-touristique au Québec}

\section{Jean Désy}

Volume 1, numéro 3, 3e trimestre 1982

Les enjeux du tourisme social

URI : https://id.erudit.org/iderudit/1080846ar

DOI : https://doi.org/10.7202/1080846ar

Aller au sommaire du numéro

Éditeur(s)

Université du Québec à Montréal

ISSN

0712-8657 (imprimé)

1923-2705 (numérique)

Découvrir la revue

Citer cet article

Désy, J. (1982). L’hébergement socio-touristique au Québec. Téoros, 1(3), 10-17. https://doi.org/10.7202/1080846ar d'utilisation que vous pouvez consulter en ligne.

https://apropos.erudit.org/fr/usagers/politique-dutilisation/ 


\section{L'hébergement socio-touristique au Québec}

par Jean Desy*

Trois éléments-clé auront surgi avec force, depuis deux ans, des pages du calendrier "socio-touristique" québécois. Pour faire image il s'agit presque d'un menu-sandwich: un vaste débat national sur la formule VVF au Québec, précédé et suivi de deux congrès majeurs du BITS, I'un ầ l'échelle mondiale, l'autre à l'échelle nord-américaine. ${ }^{\text {(1) }}$ Ces congrès, événements intenses et concentrés dans le temps, ont cette propriété de forcer la réflexion et l'action des intervenants impliqués au coeur ou même en marge du débat sur le tourisme social. IIs auront notamment été l'occasion privilégiée de faire le point sur l'hébergement touristique à vocation sociale au Québec. Ils auront fourni une tribune internationale au débat de fond engagé depuis plus de deux ans déjà par le mínistère du Loisir, de la Chasse et de la Pêche: quelles sont les possibilités et les conditions de développement de la formule Villages-Vacances-Familles (V.V.F.) au Québec? Quelle que soit la pertinence de cette question dans le contexte actuel, elle a néanmoins suscité de nombreux colloques, séminaires, documents de réflexion, articles et communications fort variés, essentiellement inspirés par le RONLO IRegroupement des organismes nationaux de loisir du Québecl.

Dans la foulée de cette effervescence, nour proposons une réflexion à la fois synthèse et exploratoire sur le thème de l'hébergement socio-touristique au Québec. Le cadre temporel de cet essai est double puisqu'il s'agira d'abord de fixer le portrait actuel de ce secteur, puis d'en esquisser très globalement l'évolution depuis ses débuts.

- Je tiens a remercier de facon toute particuliene les personnes et organirmes qui ont thoondu avec empressement a to cuellatis te donnes ricermes pour les fins de cel baticke Je ne peur tou nelois. que deplarer, sans doute de concert awo ces nombreux collaborateus $\mathrm{k}$, les taibies retomberes diectes qu aura findernent permis leur investissement de temps ef d therget, pour les ra sons, expliques plus toin.

Merci :

Andie Lauzon, de la Fédeation des agricotours du Qutbe Syive Gagnon, de la Sociphe quetbeccise du plein air Serge Belangei. du Service d hotellerie au MICT. lleman Bolduc, de la Federation quetotcoise du camping coravaning

Marcel Lemoy service de l'heherpemeat de la Socide Vacan ces Famileas.

Rene Bastien, de l'Association des camps du Cuebec. Guv Clautier, Service de lagrenent, M.A.S -Québec.

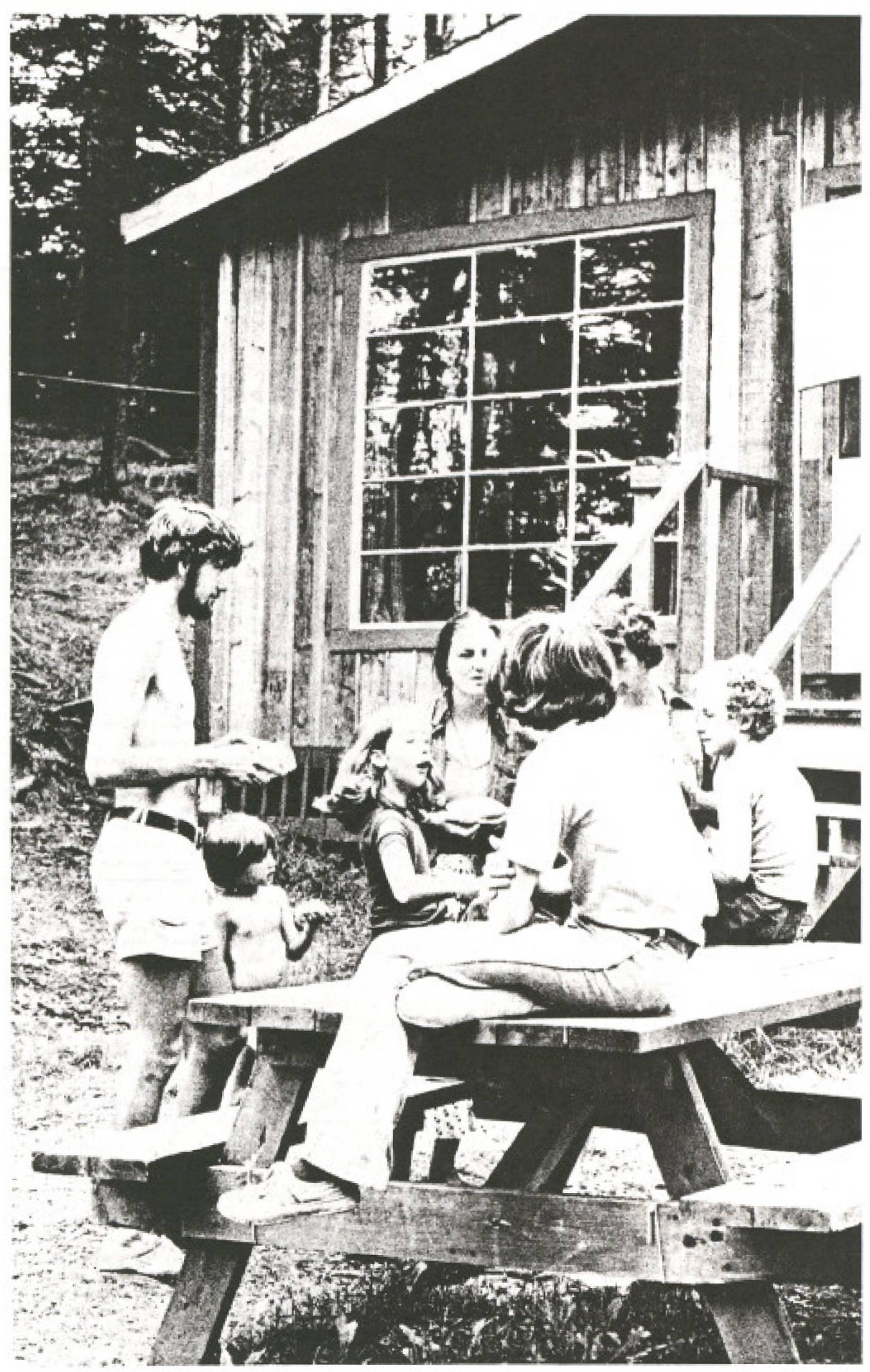


Nous appuyant sur les idées-maitresses de Bellefleur et Levasseur ${ }^{(2)}$ caractérisant le loisir et la société québecoise contemporaine. les complétant par une chronologie événementielle plus précise des faits dominants de l'histoire du Québec depuis 180 ans, nous tenterons:

1) de bien camper le profil national et régionalla de ce type d'hébergement;

2) de jeter un coup d'oeil rétrospectif sur l'évolution de ces équipements depuis leur émergence, les situant dans leur contexte sociétal et institutionnel;

3) de commenter les récents résultats du rapport gouvernemental sur la consultation des intervenants, relative à la possible implantation de la formule VVF au Québec.

1. Le dernier portrait de famille de l'hébergement socio-touristique québécois.

Soucieux de conserver un minimum de cohérence et d'homogénété entre nos don" nées et celles de la dernière analyse exhaustive du réseau d'hébergement et d'accueil du Québec, ${ }_{r}^{|4|}$ nous nous inspirerons de la typologie des equipements mises au point dans ce document. D'autre part aucune récente mise à jour globale n'ayant été effectuee par les services gouvernementaux du MLCP, nous nous baserons pour l'essentiel sur les chiffres fournis dans ce document, datés de 1978-79; à l'occasion toutefois, nous compléterons certaines données manquantes par des chiffres plus précis, fournis par les services gouvernementaux ou organismes compétents. Notre effort d'actualisation globale des chiffre s'est avéré vain en dernière analyse, en raison des nombreux trous statistiques impossibles à combler dans les délais de production impartis."

L'analyse portera donc sur le tout et les parties, c'est-à-dire d'abord sur la situation de l'équipement socio-touristique à l'échelle du Québec et ensuite sur ses variations régionales (par régions administratives). Les deux unités de mesures retenues sont le nombre d'unités d'hébergement et la capacité theorique, calculée en personnes/jour ${ }^{15 !}$. A l'occasion, nous nuancerons ces deux unités de mesure par des indices de fréquentation. mais essentiellement, I'analyse s'effectuera sur la base de données statiques. Enfin, dans le but de relativiser ces chiffres, on proce dera ă des comparaisons basées sur les données démographiques régionalisées. C'est ce que le MLCP appelle: "Indice de la distribution des équipements/établissements par rapport à la répartition de la population". "Eet indice se rapproche du taux de fonction touristique de P. Defert ${ }^{[7]}$ et il nous apparaitt pertinent de l'introduire ici, dans la mesure où l'équipement sociotouristique d'une région doit profiter au maximum à cette même population, plus encore que l'équipement touristique commercial de cette région.

"Voir tableas 1. pp. 14.15

\subsection{Les équipements}

"socio-touristiques":

un choix parmi d'autres.

Sur la quinzaine de types d'unité d'hébergement qui constituent l'éventail complet du réseau d'hébergement et d'accueil touristique au Québec, nous avons délibérément rejeté deux catégories d'établissements:

1. Les hôtels et hôtels-motels offrant le gite pour plus de 25.00 \$ par jour pour deux personnes, ce seuil financier ayant été retenu de facon arbitraire et correspondant de fait a 30 et 35 dollars pour une famile. C'est par ailleurs le taux affiché des chambres les moins couteuses de chaque hòtel.

2. Les pourvoiries de chasse et pêche, dont les prestations ne rencontrent habituellement pas les normes minimales d'accessibilité pour la majorité des vacanciers-sportifs.

D'autre part, nous avons inclus les résidences secondaires/chalets privés dans cette liste, conscient de l'importance décisive de ce type d'hébergement dans l'ofre sociotouristique totale: $98 \%$ des unités et $70 \%$ de la capacité d'accueil, hōtels y compris! On estime par ailleurs a $10 \%$ seulement le stock de chalets offerts a la location. Cette situation n'infirme pas le fait que pour des milliers de Québécois, cet équipement représente un refuge de vacances à prix encore modique, toutes proportions gardées.

Nous sommes enfin averti des buts très lucratifs poursuivis par les établissements hôteliers et la majorité des terrains de camping, voire me̊me les fermes touristiques. Si le caractère socio-touristique des équipements ne s'affirme que par le seul critère d'accessibilité financière, nous sommes d'accord: c'est peu! Mais en même temps énorme...

Retenons donc globalement dix types d'unités d'hébergement et d'accueil:

- les hôtels, hôtels/motels, maisons de chambres et autres maisons de logement inférieurs à 25.00 \$/jour/2 pers.

- les terrains de camping-caravaning

- les chalets et pavillons dans les parcs

- les chalets privés

- les fermes d'hébergement

- les résidences principales offrant l'hébergement chez l'habitant

- les résidences étudiantes des CEGEP et Universités

- les auberges de jeunesse

- les camps et colonies de vacances

- les bases de plein air.
1.2 Les grandes lignes de force à l'échelle québécoise

On aura vite compris l'inutilité de longs commentaires sur la répartition des unités selon les types, puisque $98,3 \%$ des équipements sont des chalets... Par contre, la grande logeabilite des mille terrains de camping équilibre quelque peu la répartition de la capacité théorique d'accueil: ces terrains. peuvent héberger près d'un vacancier sur quatre, rabaissant à moins de trois sur quatre $(70 \%)$ le nombre de villégiateurs sur le total d'hébergés. Hôtels et camps de vacanges peuvent accueillir respectivement un vacancier sur $40(2,5 \%)$. Au total, les quelque 170,000 unités accueillent théoriquement plus d'un million de personnes sur le territoire québécois. En comparant cette capacité à celle de la population résidente, on obtient un "taux de fonction sociotouristique" de 16.7, movenne nationale quion confrontera plus loin à celle des régions.

\subsection{Une répartition étonnante}

et contrastée à l'échelle des régions. Sans conteste, la région de Montréal tranche nettement sur toutes les autres, avec $46 \%$ des unités et $44 \%$ de la capacité d'accueil. Québec et l'Outaouais suivent de loin pour ces deux variables, sur un relatif pied d'égalité (de 13 à 16\%). Puis la Mauricie, avec 8 et $9 \%$, la Gaspésie-Bas du Fleuve. la Sagamie et l'Estrie oscillent autour de 4 à $6 \%$. On ne s'étonnera pas des basses performances de la côte nord et du Nouveau-Québec.

Mais ce tableau déséquilibré se transforme bizarrement avec l'introduction du taux de fonction socio-touristique: l'obésité démographique de Montréal fait chuter la région sous la moyenne nationale, en 7ième position, $\mathrm{tf}$ (st) $=13$, tandis que le faible poids. de I'Outaouais, allié à sa bonne capacité d'accueil, place loin en tête cette région sous ce rapport tf (st) $=52$ !

L'Estrie se distingue ensuite, seule de son rang 27 , immédiatement suivie de trois régions périphériques: Gaspésie, Sagamie et Mauricie, 21 a 23; puis loin derrière, sous la movenne nationale viennent la Côte Nord et I'Abitibi-Témiscamingue, 11 et 12 .

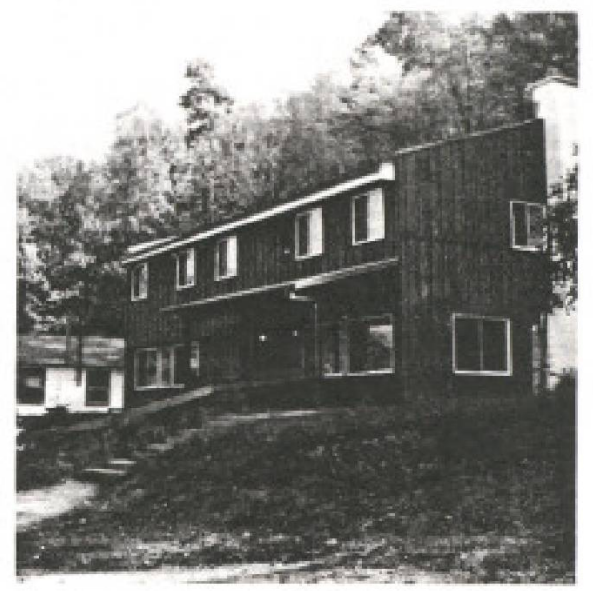


1.4 Quelques traits complémentaires, par types d'hébergement et par région.

\section{- Les équiperments hötehiers}

II nous semble intéressant de nous attarder sur la nouvelle physionomie de cet intervenant, en forte évolution depuis quelcues années, la crise économique ayant un impact majeur sur l'évolution du coût des chambres, de même que sur le profil de la structure d'accueil. Signalons d'abord que seulement 800 établissements hôteliers du Québec, soit deux hôtels sur cinq $(41 \%)$, offrent 12,000 chambres a prix abordables, soit une capacité de 26000 personnes/jour. ce qui représente le 1/5 des chambres disponibles. Par contre, à l'autre bout de l'échelle, 377 hôtels, soit un hôtel sur cing $(19,2 \%)$, offrent 31000 chambres de plus de $40.00 \$ \sqrt{j} / 2$ pers, soit une capacité thérique de 67300 personnes/jour, ce qui repré. sente plus de la moitie des chambres dis. ponibles au Québec $(51,6 \%)$ !

Examinons le détail des régions: Les régions de Montréal et Québec offrent chacune le quart de l'offre d'unités et de chambres (à moins de $25.00 \$ / / .2$ pers.l, soit au total, la moitié de l'accueil hôtelier à prix modique au Québec. Sagamie et Cote Nord se situent à l'extrême bas de l'échelle sur les deux plans, inombre de chambres et nom. bre d'hôtels), mis a part le Nouveau-Québec (néantl. La Gaspésie-Bas-St-Laurent, région de tourisme, s'inscrit pour 13 à $15 \%$ de l'offre modique, les quatre autres régions oscillant entre 5 et $11 \%$ de l'offre.

Par contre, c'est la région de Montréal qui domine nettement l'offre de chambres "Iuxueuses" ( $40.00 \$ / j_{2} / 2$ pers.) avec deux hôtels sur cinq du parc hôtelier québécois et plus de la moitié des chambres disponibles. La région de Québec suit avec une chambre et un hôtel sur cinq dans cette catégorie. Au total, pour ces deux régions (sinon ces deux villes): $3 / 5$ des établissements hôteliers "luxueux" et les $3 / 4$ des chambres luxueuses du Ouébec... Le poids cumulé de ces deux régions déséquilibre la répartition régionale de la movenne québée coise dans cette catégorie lun hôtel sur cinq et une chambre sur deux); alors que les autres régions balancent entre 2 et $8 \%$ de l'offre inaccessible à la majorité. Une conclusion s'impose: le double mouvement de concentration spatiale des hôtels luxueux des chaines internationales et de diminution de l'offre raisonnable se poursuit inexorable ment. Une percée encourageante de la Société Vacances-Familles auprès de ces chaines et de nombreux hôttels et auberges (70 au total), permet toutefois une fréquentation accrue de ces ettablissements, notamment pour la clientèle familiale, au moyen de rabais substantiels surtout en morte saison.
- Le camping-caravaning.

C'est encore le monopole des rives sud et nord de Montréal, oủ se concentrent le tiers des terrains du Québec et pouvant accomoder deux campeurs sur cinq. Se distinguent les régions de Québec $(1 / 5$ des terrains et de la capacité), et de la Mauricie (11 et $13 \%$ ).

- Les résidences secondaires/chalets prives.

La longue tradition de villégiature des Laurentides au nord de Montréal assure une nette suprématie de la région 06 sur le reste du Québec: $46 \%$ de l'offre s'y concentrent. Vient ensuite |'Outaouais $(16,4 \%)$ qui récolte pour ce type d'équipement l'essentiel de sa capacité d'accueil, lui accordant le plus haut taux de fonction socio-touristique du Qué bec. Le réseau de la S.V.F. soumet à ses membres un nombre croissant de chalets privés offerts à la location.

\section{- Les fermes d'hébergement.}

Emergeant depuis moins de dix ans dans le paysage québécois, notamment au sein du réseau des Agricotours et de VacancesFamilles, ces fermes offrent egalement pavillons autonomes, emplacements de camping et gites du passant (le système "bed and breakfast $\left.{ }^{\prime \prime}\right)$. On les retrouve pour le tiers en Gaspésie-Bas du Fleuve-lles, pour le cin= quième dans la région de Québec, et en bonne quantité en Sagamie et dans les régions rurales montréalaises (de 12 a $16 \%$ ). $C^{\prime}$ est un réseau fluctuant, mais en progression et ajustements réguliers.

\section{- Les résidences principales chez Thabitant.}

Formule encore marginale, mais prometteuse en ces temps difficiles, on compte néanmoins 315 unités au Québec pour 630 places. Ce sont des villages commes Desbiens ou Chambord au lac St-Jean, en collaboration avec SVF, qui ont innové en ce domaine depuis cinq ou six ans: le tiers de l'accueil s'y concentre, le quart à Montreal et le cinquièrme à Québec.

\section{- Les résidences étudiantes.}

L'ère du béton des années 60 dans le domaine de l'éducation au Québec a sus. cité bien des remous écologiques, et a notamment permis un heureux recyclage. soit leur utilisation à des fins d'hébergement populaire. Quelque 26 résidences étudiantes de collèges et universités peuvent théoriquement accueillir chaque jour de l'été, environ 8500 personnes. Le tiers des chambres se concentre bien sûr à Montréal, mais un autre tiers à Québec et plus de $16 \%$ dans I'Estrie.

\section{- Les auberges de jeunesse.}

Ces auberges connues depuis plus de quinze ans au Québec, sont au nombre de 35 et peuvent offrir le gitte à plus de 2000 jeunes par jour. Trois concentrations surgissent nettement: régions de Montréal, Qué bec et Gaspésie-Bas-St-Laurent, avec des pourcentages voisins, totalisant à elles trois $75 \%$ de la capacité d'accueil du Québec à ce chapitre.

\section{- Les camps et colonies de vacances.}

C'est l'écrasante suprématie de la région montréalaise: 3 camps sur 5! La capacité d'accueil totale pour le Québec [27 000 personnes) rivlaise avec celle des hötels à prix modique, mais avec un nombre beaucoup plus faible d'unités 233 camps vs 808 hôtèlsi. Depuis un peu plus de dix ans, le Mouvement québécois des camps familiaux procède également à un recyclage structurel et idéologique de cet héritage clérical et c'est maintenant environ 30 groupes familiaux qui louent ou gèrent un camp familial, au profit de plus de mille familles québécoises de milieu populaire.

\section{- Les bases de plein air.}

Une soixantaine de bases ont pignon sur rang au Québec, dont une trentaine offrent l'hébergement à un maximum théorique de 5300 personnes, les deux tiers d'entre elles dans des constructions en dur, le dernier tiers en camping. La région de Montréal se distingue avec $44 \%$ des places et des unités, celle de Québec suivant de loin avec le cinquième, celle de l'Estrie avec $16 \%$ des places, celle de la Sagamie avec $11 \%$.

La clientèle se recrute surtout parmi les adolescents et les jeunes couples, mais une bonne demi-douzaine de bases ouvrent depuis quelques années à la clientèle familiale.

Voilà donc, brossé à grands traits, le tableau fort diversifié de l'hébergement sociotouristique du Québec. II semble donc se dessiner:

1) une diminution importante de la part de I'hótellerie financièrement accessible, mais egalement un effort timide des hótels luxueux auprès d'une clientèle familiale par une réduction modulée des tarifs;

2) un plafonnement prévisible du parc de chalets privés, mais aussi une ouverture encourageante à une accessibilité plus grande par le biais de la location;

3) une progression désormais plus moderée du camping-caravaning, I'accent étant mis sur la modernisation, sur son adaptation, plus coûteuse cependant, au caravaning:

4) une poussée et une diversification des nouvelles formes d'hébergement sociotouristique nées de la dernière décennie, quelquefois récupérées des équipements plus traditionnels (colonies de vacances) ou plus institutionnels (résidences d'étudiantsl.

A ce propos, il semblerait pertinent de visionner le cheminement historique et sociétal de ces équipements pour en comprendre davantage la dynamique à venir. C'est l'objet du point suivant. 


\section{Une croissance, effet et reflet} de celle de la société québécoise. Dans leur dossier-choc sur le passé, le présent et l'avenir des pratiques de loisir au Québec ${ }_{r}^{(9)}$ Bellefleur et Levasseur soutiennent essentiellement "que nos institutions en loisir sont largement touchees par des normes et des valeurs dominantes dans notre modèle social". "10i" "Dans ce système oú les hommes sont egaux en droit mais inégaux en fait, le loisir repro. duit a son niveau la stratification sociale, les disparités et inégalités économiques et culturelles que lon observe partout alleurs. Au plan des institutions, le modele libéral ne laissant à l'Etat qu'un rôle résiduel, il est manifeste que ce sont les initiatives priveses les plus dynamiques qui ont cred les premieres organisations et structures en loisir au Québec. "थl1" S'opposent ou se superposent à ce modèle libéral, le modèle technocratique, "pouvoir base sur /information et la reconnaissance scientifiquerfl|z| et le modèle participationniste -où l'on valorise d'une facon dominante la participation des citoyens par le truchement de leurs groupes structurés, à l'orientation du développement sociétal. Sur cette base, nous tenterons d'échafauder une charpente rudimentaire d'approche "socio-temporelle", vaguement systémique, joignant donc quelques éléments sélectionnés sur une échelle du temps horizontale, divisée par des événements marquants depuis 1850 jusqu'à l'actuelle décennie. * Ces élements sont:

1. les classes sociales dominantes qui se sont succédées et qui ont marqué de facon déterminante:

2. les phénomènes socio-politiques spatiaux et technologiques décisifs de ces périodes, causes ou conditions de 3 .

3. les formes de loisir et d'hébergement associés. 113 ?

Nous ne commenterons que superficiellement les propositions de ce tableau trop vite ebauché, mais suffisamment étoffé. croyons-nous, pour suivre dans ses grandes lignes l'évolution du réseau d'hébergement, socio-touristique actuel et en devenir au Québec.

Les débuts de l'ère touristique au Québec semblent reposer sur le capital de temps et d'argent accumulế par l'élite traditionnelle canadienne francaise de l'époque. Ils se matérialiseront d'abord dans une station touristique du Bas du Fleuve, Kamouraska, mais aussi dans des localités voisines de Québec et Montréal (Cap Rouge, Beauport, pentes du Mont-Royall. Le tourisme itinérant américain, britannique ou canadien a également envahi le Bas Canada par diligence ou bateaux vapeurs, provoquant par le fait mëme l'éclosion d’une hôtellerie adaptée ou à tout le moins, consolidant le réseau d'auberges existant. Mais la véritable expansion de la villégiature, telle que connue simultanement en Europe, c'est après 1850 qu'on la connut, grâce à l'accroissement des

- Vair tablesu 2 . 16 richesses et de l'influence de la bourgeoisie anglophone du continent, maitresse de l'industrialisation naissante au pays. La station touristique classique, (La MalbaiePointe-au-Pic, Cap-à-l'Aigle, St-Irénée, Cacouna, Métis, Tadoussac, Roberval) a donc connu son apogée, émaillée d'hôtels prestigieux et de villas ronflantes, souvent occupees tout l'été, tous lieux accessibles par chemin de fer. A Montréal, la villégiature s'installa sur les bords du fleuve surtout, et avec le temps, tout autour de l'ile. Vers la fin du 19ième siècle, les Laurentides et les Cantons de l'Est s'ouvrent à ce même type d'équipement touristique. Si les loisirs touristiques du peuple urbanisé de Montréal et Québec peuvent aussi prétendre à l'existence par des excursions de courte durée dans les environs, ils ne semblent pas géné rer de lieux d'hébergement à leur portée et à leur mesure. Le tourisme religieux a possiblement ouvert plus de portes sur ce plan. Si l'on songe aux foules qu'attirait déjà SteAnne-de-Beaupré... $(14)$

C'est d'ailleurs le clergé qui bâtira aux fils et filles d'ouvriers de Montréal, la première colonie de vacances en 1912, "Les Grèves de Contrecoeur- "afin de protéger les enfants de Montréal, et phs particulierement les enfants de familles paurres, contre les dangers de toutes sortes auxquels ils sont exposes durant les vacances $d^{\prime} e t e^{\text {nt5 }}$ On y accueillera plus 2000 petits "Colons" vers 1950. On connait le succès que remportèrent ces colonies de vacances gérées par le clergé et dont le nombre a atteint plusieurs centaines à travers le Québec. La Révolution tranquille y a opéré une laicisation porteuse d'un nom et d'un esprit nouveaux: les 233 camps de vacances diversifièrent leurs clientèles et s'adaptèrent même aux familles.

Mais un autre hébergement de loisir se transforme progressivement après la première guerre, automobile, urbanisation et revenus aidant: c'est le chălet de villégiature. On comptera plus de 28000 chalets en $1941^{116 !}$, dont beaucoup agglomérés dans et autour de centres de villégiature. La véritable expansion s'est produite à partir des années soixante, rejoignant toutes les régions du Québec, (concentration en bordure des Laurentides et Appalachesi et toutes les classes de la société, mais en particulier les couches moyennes et populaires: "conduites d'imitation" des strates moyennes dominées par les strates supérieures? ${ }^{217)}$ Sans doute ce processus de stratification sociale en loisir, soumis par Touraine, joue-t-il pour beaucoup. Mais bien d'autres facteurs encore, dont ceux qui ont déclenché les premiers déferlements de campeurs après la deuxième guerre: plein air et sociabilité, auxquels se rajoute un attrait pour le nomadisme que partage moins le villégiateur. " Le phénomène est devenu "de masse", là encore au cours de la décennie 60 , et ce type d'équipement socio-touristique, du moins dans sa version "soft-ware". a sans doute été et demeure encore le fer de lance de l'accessibilité maximale aux vacances pour une majorité, l'accession à la propriétế automobile remplissant une seconde condition de départ.

De son côté, l'hôtelletie d'après-guerre a su accueillir un nombre croissant de vacanciers, notamment dans ces sordides "cabins" aujourd'hui disparues, dans des hỏtels, motels et auberges désireux de rendre accessible le rêve des prestigieux hôtels bourgeois d'une époque révolue. Nous avons déjà établi que le seul critère d'inclusion de I'hótellerie commerciale dans ce réseau d'hébergement socio-touristique était son accessibilite financiere aux clientèles populaires et moyennes, ou des clientèles spécifiques (familles). L'essor comparative: ment plus prononcé - et encouragé - des luxueux hôtels de chaînes multinationales (Sheraton, Hilton, Holiday-Inn...) au cours des deux dernières décennies, a fait chuter le stock global de chambres bon marché.

Enfin, les clubs de chasse et peche et les pourvoiries, situés en périphérie du Québec de base et des régions ressources, créés depuis plus de cent ans par les bourgeoisies possédantes, ont bien peu contribué à l'enrichissement du réseau socio-touristique. Jusqu'à la création des ZEC à la fin de la décennie 70 , les clubs privés accaparaient $87 \%$ du territoire de chasse et de pëche accessible par voie de terre et le membership ne touchait guère plus de $5 \%$ des populations régionales. Les chiffres concernant le réseau de camps sont rares, mais sans doute décevants dans le cadre de nos préoccupations. Les projets de lotissement du MER (Energie et Ressources) sur les terres publiques vont possiblement modifier ce tableau. Quant aux pourvoiries, leur très faible accessibilité financière ne mérite pas de commentaires. Bref, les années d'aprèsguerre ont connu:

1) une expansion marquée de certains hébergements touristiques bourgeois prestigieux -hốtellerie, villégiature de résidences secondaires, camps de chasse et pêche à la limite-,

2) une forte poussée des hébergements de vacances charitabies du clergé lcolonies de vacances),

3) une croissance rapide d'un mode commercial mais populaire d'hébergement. le camping. C'est tout au long des vingt dernières années que campings et chalets vont fracasser des records de popularité, mais toujours sous le signe de l'entreprise privée ou individuelle. Car si la révolution tranquille des années 60 va faire bouillonner le Québec dans un bain de mesures sociales, le loisir lui, va y échapper: gouvernement comme syndicats ont trop d'urgences, trop de retards vitaux à rattraper. Ce ne sera qu'au cours de la décennie suivante, qu'apparaîtront les premières implications gouvernementales structurées: 
- Subventions des camps familiaux, en émergence.

- Subventions d'auberges de jeunesse et de bases de plein air.

- Soutien à l'agro-tourisme.

- Création de la Société d'Aménagement de l'Outaouais (S.A.O.) qui contient en germe les éléments de la formule VVF.

- Création des ZEC.

- Création d'un ministère du Loisir, après une vaste consultation.

- Mise sur pied d'un comité interminestériel sur la faisabilité des VVF au Québec.

- Multiplication des "missions" gouvernementales sur la formule VVF en France.

Concurremment, le monde du loisir sociotouristique s'organise et pousse aux réformes, provoque cet intérét:

- Le GRVS. IGroupe Ressources-Vacances Sites) soutient techniquement des groupes populaires de Montréal à la recherche des lieux et d'équipements de vacances conformes à leurs besoins;

- Ces groupes s'autonomisent et deviennent le Mouvement québécois des camps familiaux. (1981)

- La Société Vacances-Familles ouvre un front large de tourisme social par la mise en place d'un réseau de plus en plus diversifié d'équipements d'accueil Ichalets, hốtels, fermes, résidences étudiantes, résidences principales, auberges). (1971-82)
- La CLO IConfédération des loisirs du Québec) puis le RONLO. (Regroupement des organismes nationaux de loisir du Québec) multipient les interventions, les propositions pour un tourisme social actif. (1970-82).

- Création du Groupe de ressources en tourisme social - (1982).

- Création des Agricotours, en collabora. tion avec I'UPA et le ministère de I'Agriculture.

- Ouverture des institutions d'enseignement à l'accueil touristique.

- Effervescence de nombreux intervenants régionaux désireux de concrétiser la formule VVF dans leurs régions.

En fait, le "loisir touristique" a eu sa révolution tranquille au cours des années 70. A-telle des chances de se prolonger dans les annees 80 et de voir le Quebec enrichi d'un réseau socio-touristique convenable en 90 ? Le récent bilan de la consultation sur la formule VVF au Québec ${ }^{19}$, fournit quelques pistes.
3. Vers une consolidation du réseau actuel, avec ou sans VVF?

Depuis vingt ans déjà que missions et délé gations québécoises en France allaient s'inspirer du modèle VVF, dans une perspective d'application québécoise, sans accoucher de quoi que ce soit d'autre que de rapports sans lendemains: il était temps de passer aux actes. Le Livre blanc sur le loisir au Qué bec en fournit le premier jalon: "Ainsi le gouvernement appuiera et stimulera le développement d'un réseau de type "villages-vacances-familles" (VVF) et la consolidation d'auberges de jeunesse et de bases de plein air" nistériel se forme en janvier 1980 , qui soumet d'abord une proportion (avril 81) et qui recueille ensuite les réactions du milieu du printemps à l'automne suivant. Le rapport cité (juillet 82 ) fait donc état de la rétro-action de quelque 26 organismes, contactés en deux étapes Imémoires écrits et séances publiques de consultation). II y aura donc eu un vaste remue-méninges dans le monde du plein-air et du tourisme social, que Bellefleur et Levasseur situeraient peut-être dans le modèle social "de participation". malgré l'origine technocratique du processus... Seule la suite et fin de l'operation statuera sur ce point.

Mais jetons plutôt un coup d'oeil sur les conclusions du rapport, qui établissent les points de convergence et de divergence entre le rapport OPDQ-MLCP et les avis du milieu, et qui tranchent finalement sur la

Capacité du réseau d'hébergen par régions admi

\begin{tabular}{|c|c|c|c|c|c|c|c|c|c|c|c|c|c|c|c|c|c|c|c|c|c|c|c|c|}
\hline \multirow{3}{*}{ Rdgiens administratmes } & \multicolumn{12}{|c|}{ Atseau 1} & \multicolumn{12}{|c|}{ Atseau 2} \\
\hline & \multicolumn{4}{|c|}{ 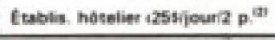 } & \multicolumn{4}{|c|}{ Twrrains de eamping c carav. } & \multicolumn{4}{|c|}{ Chuitets of parilloms pares } & \multicolumn{4}{|c|}{ Ritsidences second ichainsts } & \multicolumn{4}{|c|}{ Fermes d hebergement } & \multicolumn{4}{|c|}{ Atesidencess principsins } \\
\hline & A & B\% & $\mathrm{c}^{\mathrm{at}}$ & ow & $\mathbf{A}$ & as & $c^{+4}$ & D* & A & os & $\mathrm{c}$ & D* & A & B* & $\mathrm{c}^{\mathrm{H}}$ & D* & A & $18 \%$ & $c^{+\infty}$ & $D=$ & A & ex & c & E \\
\hline On Gasposie Bes Saint Laureat & 100 & 13.1 & 5e00 & 14.6 & $10 \%$ & 11 & $17 \pi 9$ & 7 & 66 & 20,4 & 392 & 18.5 & 6142 & 3.7 & 27393 & 37 & so & 34,3 & 278 & 33,3 & 28 & 9 & 56 & \\
\hline b2 Sagamie ISep Lae St Jeani & 24 & 30 & 17233 & 4.7 & 69 & $?$ & 18580 & $s$ & 29 & g & 149 & 7,1 & 3463 & 5,1 & 37745 & 5.1 & 24 & 16.4 & 115 & 13.7 & 104 & 3 & 212 & \\
\hline 03 Qutbec & 209 & 25.8 & 5428 & 245 & 212 & 21 & 42550 & 18 & 97 & 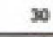 & Tol & D.3 & $2069 ?$ & 12.4 & 92309 & 124 & 29 & 199 & 174 & 20.8 & 6 & 19 & 120 & \\
\hline of Monicie & 63 & 6.4 & 1994 & 7.6 & 106 & 11 & 39741 & 13 & 19 & 59 & 307 & 14,6 & 17962 & 7,8 & 57900 & 7,8 & B & 5,5 & 49 & 5.9 & is & B & so & \\
\hline OS Evtrie & 37 & 4,6 & 1148 & 4,4 & 53 & 5 & 10020 & 4 & - & - & - & - & iones & 6.3 & 4609 & 6.3 & 6 & 4.1 & 34 & 4.1 & 12 & 4 & 24 & \\
\hline 106 Monurtal & 201 & 24.8 & 6111 & 23.4 & 311 & 31 & 94201 & 39 & 63 & 19.5 & 153 & 7,3 & 76701 & 46.0 & 342006 & 46.0 & 20 & 137 & 104 & 12.5 & $\pi$ & 24 & 150 & \\
\hline कif Outanuais & B) & 10,8 & 2166 & 8,3 & 73 & 7 & 16184 & 7 & 27 & 8.4 & 230 & 10.9 & 27477 & 16.5 & 122547 & 16.5 & 5 & 34 & 29 & 35 & 7 & 2 & 14 & \\
\hline of Abinibi Tamiscamingue & 46 & 5,7 & 2146 & 0.2 & a1 & 4 & 613 & 2 & - & - & - & - & 1630 & 1.0 & 770 & 1,0 & 4 & 27 & 52 & 5.7 & 2 & 1 & 4 & \\
\hline os cone Nond & 30 & 37 & 1106 & 4,2 & 19 & 2 & 3660 & 2 & $n$ & 6,8 & 170 & B. 1 & 2002 & 1,2 & as: & 1.2 & - & - & - & - & - & - & - & \\
\hline 10 Noumatu Qutbec & + & $=$ & $=$ & $=$ & 7 & 1 & $102 ?$ & it & - & - & - & - & - & - & - & - & - & - & - & - & - & - & - & \\
\hline Quabesc itrotali & wat & 99 & 2614 & $\$ 9.9$ & sea & 1000 & 247365 & 100,0 & 383 & 100,0 & 2102 & 999 & 166563 & 100,0 & 742871 & 100,0 & 146 & 1000 & sas & 100.0 & 315 & 1000 & 630 & $\pi$ \\
\hline Part relative pht hu total quet. & & 05 & & 25 & & 06 & & 22.9 & & $a, 2$ & & 0,2 & & 95.3 & & 70.2 & & 0,1 & & 0,06 & & 0.2 & & $i$ \\
\hline
\end{tabular}

A Nombre d'unites d'hébergement en valeur absolue

B \% du nombre d'unites d'hebergement par rapport à l'ensemble des uh, du Quebec, pour cente categorie

C capacite therique d accueil, calcule en nombre de per sonnes/jour

D \% de cette capacité theorique d'accueil par rappori à celle du Quebec
Réseau 1:

Les etablissements privés exploités commercialement et les etablissements publics pour voyageurs et touris. tes/vacanciers

Réseau 2 :

Les etablissements prives exploités occasionnellement a des fins commerciales pour touristes vacanciers
Réseau 3:

Les établissements publics occasionnellement access. bles aux touristes vacanciers en dehors de leur période d'utilisation lièe à leur finalité

Réseaบ 4:

Les etablissements geres par des organimes sans but lucratif. 
volonté d'expérimentation de la formule par le MLCP:

"Les points fondamentaux de convergence avec le rapport $O P D Q-M L C P$

Il est admis que les équipements et les services nécessaires pour pratiquer le loisir touristique en famile au Quebec sont inadequats et insuffisants.

Les vacances familales en collectivité sont percues comme une formule qui présente de /intérêt.

On admet que les formules à privilegier pour satisfaire les besoins en loisir touristique des familles devront mettre /accent sur lanimation, la participation, la polyvalence voire la multi-affectation des equipements.

On considere que FEtat devra apporter une contribution financière importante au niveau des immobilisations et méme prévoir une aide a la personne pour que puisse se développer la formule de type V.V.F. Cette aide a la personne ne devraít pas etre exclusivement orientée vers la fréquentation des V.V.F. mais également etre accessible aux divers réseaux existants.

On estime enfin que le MLCP devra assumer des responsabilités de planification et d'aide à la gestion.
Les principales réserves exprimées Les principales reserves exprimees au cours de la consultation concemant le rapport do comite conjoint OPDQ - MLCP et la specificite du sujet soumis a la consultation.

En regard du rapport

du comité conjoint OPDQ-MLCP

La majorite des intervenants considere que le reseau quebelois de loisir touristique en regard de son potentiel de developpement par rapport a / hébergement familial aurait été sous-évalué et aurait dù faire /objet d'un traitement plus adéquat.

\section{Le concept propose}

On a reproché une référence trop exclusive à des modeles etrangers. On souhaite par ailleurs un concept de willage de vacances qui soit mieux enraciné au contexte et aux moeurs québecois tant au niveau de la pratique des vacances qu'au niveau du développement des equipements, voire de lappellation même du concept.

Au plan du développement et de la gestion. surtout dans une perspective d'experimentation, la proposition formule par le comite conjoint $O P D Q$ - $M L C P$ de créer une corporation de niveau national n'est pas retenue.

En regard de la spécificité du sujet soumis à la consultation

Une majorite d'intervenants auraient souhaité qu'au lieu de faire porter une consul- tation sur une formule d'hebergement collectit, l'Etat les consulte au préalable sur une proposition plus globale (re: une politique du tourisme sociall qui aurait abordé les questions de lacces aux vacances et aux loisirs touristiques pour les familles ainsi que la question du temps /ibre sans oublier pour autant la consolidation des equipements et des services existants.

\section{L'experimentation}

Nonobstant les réserves exprimées ci-haut et dans la mesure ou le $M L C P$ souhaiterait aller de l'avant en cette matière, une majorité d'intervenants considererait préférable une phase d'expérimentation de la formule V.V.F., notamment du village éclate, avant toute decision definitive: "all

Tout en étant conscient de la formulation particulièrement étudiée des oppositions manifestées lafin d'éviter les mises en relief trop accusées), un observateur impartial des. consultations et de leur consignation peut porter un jugement positif sur ce compterendu. Toutefois, on pourra sans doute discuter de l'interprétation apportée à la volonté collective ferme d'une expérimentation de la formule VVF, si l'on étudie de près l"annexe 2, colonne 4.2.1. II est toutefois juste de signaler que peu d'intervenants rejetteraient l'expérimentation du modèle de "village éclaté", i.e. "un ensemble d"hébergements varies, issus du milieu d'accueil, dispersés sur un territoire rural englobant une ou plusieurs municipalité...

eau 1

vent socio-touristique au Québec

aistratives - 1978 (1)

\begin{tabular}{|c|c|c|c|c|c|c|c|c|c|c|c|c|c|c|c|c|c|c|c|c|c|c|}
\hline \multirow[b]{3}{*}{ s } & \multirow{2}{*}{\multicolumn{4}{|c|}{$\begin{array}{c}\text { Rtsesu } 3 \\
\text { Residencess Gudiantes }\end{array}$}} & \multicolumn{12}{|c|}{ Pesseau 4} & & & \multirow{3}{*}{ 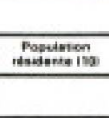 } & \multirow{3}{*}{ 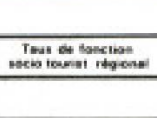 } & \multirow{3}{*}{ Asmo: } \\
\hline & & & & & \multicolumn{4}{|c|}{ Autargen do jounteise } & \multicolumn{4}{|c|}{ Cumper at calsnies de ver } & \multicolumn{4}{|c|}{ Beseses de plein-air } & \multicolumn{3}{|c|}{ Totat } & & & \\
\hline & A & 64 & c & D* & A & $8 \%$ & c & $\mathrm{DO}_{\mathrm{n}}$ & A & 10\% & $\mathrm{c}$ & D\% & A & $8 \%$ & $\mathrm{C}^{\text {is }}$ & $0 \%$ & A & $\mathrm{B} \%$ & Dow & & & \\
\hline 9 & 3 & 11,5 & 317 & 37 & 7 & 20 & 450 & 20,6 & 5 & 2.1 & 601 & 2,3 & 4 & 6,7 & $3 n$ & 7,1 & 651: & $3.8 \quad 60 \%$ & $4, \pi$ & 22750 & 2.4 & or \\
\hline 13 & 2 & 7,7 & 450 & 5.3 & 3 & 8.6 & 165 & 7,6 & 10 & 4,3 & $m$ & 3 & 5 & 8.3 & 583 & 11,1 & 8735 & $5,1 \quad 6038$ & 5,8 & 291,45 & 20,7 & 92 \\
\hline 9 & 6 & 23,1 & 2894 & 34 & 7 & 20 & 699 & 24.7 & $\$$ & 15 & 3212 & 12 & 13 & 21,7 & 1000 & 19 & 21365 & $12,6 \quad 149927$ & 14.2 & $1,006.020$ & 14.9 & as \\
\hline 8 & 2 & 7,7 & we & 2.4 & 2 & $\$ 7$ & 160 & 7,3 & 17 & $T, 3$ & 1431 & B,4 & 6 & 10 & 10 & 2.6 & $192 x$ & $7,8 \quad 9506$ & 69 & 427,708 & 22 & ins \\
\hline 4 & 4 & 15.4 & 1400 & 46.3 & 2 & 57 & 84 & 38 & 14 & 6 & 1390 & 5.2 & 4 & 6,7 & 850 & 16.2 & 10601 & $63 \quad 62242$ & $\$, 9$ & 229,160 & $n 2.2$ & 05 \\
\hline is & 7 & $\mathbf{x . 9}$ & $2 \mathrm{H} 30$ & $x$ & A & 22.8 & 615 & 28 & 129 & 5,4 & 16590 & 0.3 & 19 & 31.6 & 2145 & 40.8 & 7753 & $45,8 \quad 46450$ & 4.9 & 3607,394 & 12.9 & $\alpha$ \\
\hline 2 & - & - & - & - & - & - & $=$ & $=$ & 14 & $\theta$ & 1960 & 73 & - & - & - & - & 27690 & $16,4 \quad 143120$ & 13.5 & $276,4 \mathrm{Ba}$ & 51., & 07 \\
\hline 1 & 1 & 3.8 & 350 & 4,9 & 1 & 28 & 35 & 1.5 & 6 & 2.6 & 520 & 2 & 5 & 10 & Bo & 1,5 & 1737 & $1,1 \quad+6600$ & 10 & 147, aras & 11,2 & की \\
\hline & 1 & 3.8 & 100 & 1,2 & 5 & 14 & 138 & 6.3 & 3 & 1.3 & 142 & 05 & 3 & 5 & 90 & 1.7 & 2005 & $1.2 \quad 14341$ & 1.4 & 117.210 & 12,2 & 09 \\
\hline & - & - & - & - & - & - & - & - & - & - & $=$ & - & - & - & - & $\ldots$ & 7 & - 1027 & a.1 & 19390 & 5.2 & 10 \\
\hline 0 & 76 & 99.9 & 8555 & 100,0 & $x$ & 99,6 & 2106 & 99.9 & 239 & 10010 & 26607 & 1000 & 60 & 1000 & 5261 & 1000 & 16950\% & 100.11667661 & 1600 & 6.360 .602 & 16.7 & Outcent ionsil \\
\hline$x$ & & 0,01 & & 0.8 & & 0.02 & & 0.2 & & $a, 1$ & & 2.5 & & 000 & & 0.5 & & 1000 & 1000 & & & \\
\hline
\end{tabular}

11) Sauf indications contraires

(2) Les ettablisements pour wovageurs et touristes vacanciens; hotels, hotelsimotels, maisons de cham bres, autres maisons de logement, de catégorie a) 5 pignons, aftrant le gite pour moins de $\$ 25.002$ pers jour. Compilation spectale du service de tho tellerie du MICT, 1981 )
(3) Sur la base du nombere de chambres offertes, mul tiplie par le coefficient de 2.17 persich.

(4) Sur la base de 3.6 persiemplacement

(5) Sur la base de 4.45 persichalet

(6) Donness actualiseas de 1982, par Agricotour. Types. d'unites dihebergement compris: maisons de ferme. chalets en location, et gites du passant.
7) Otfrant I'hébergoment chez l'habitant

(6) Dans les CEGEFs et Universités.

c9) Sur la base de 3.3 persiemplacement de camping Isil $y$ a lieul.

[10) D apress une estimation - 1978- de l'annuaire du Quebec $1979 \cdot 80$ (tableau 11 et carte 1$)$

N.B. Certains chiffres apparaissent inexacts IEx. Rtgion 02: plutót 270,000 que 291,000 
Tableau 2

Évolution de l'hébergement touristique et socio-touristique au Québec

$1800-1982$

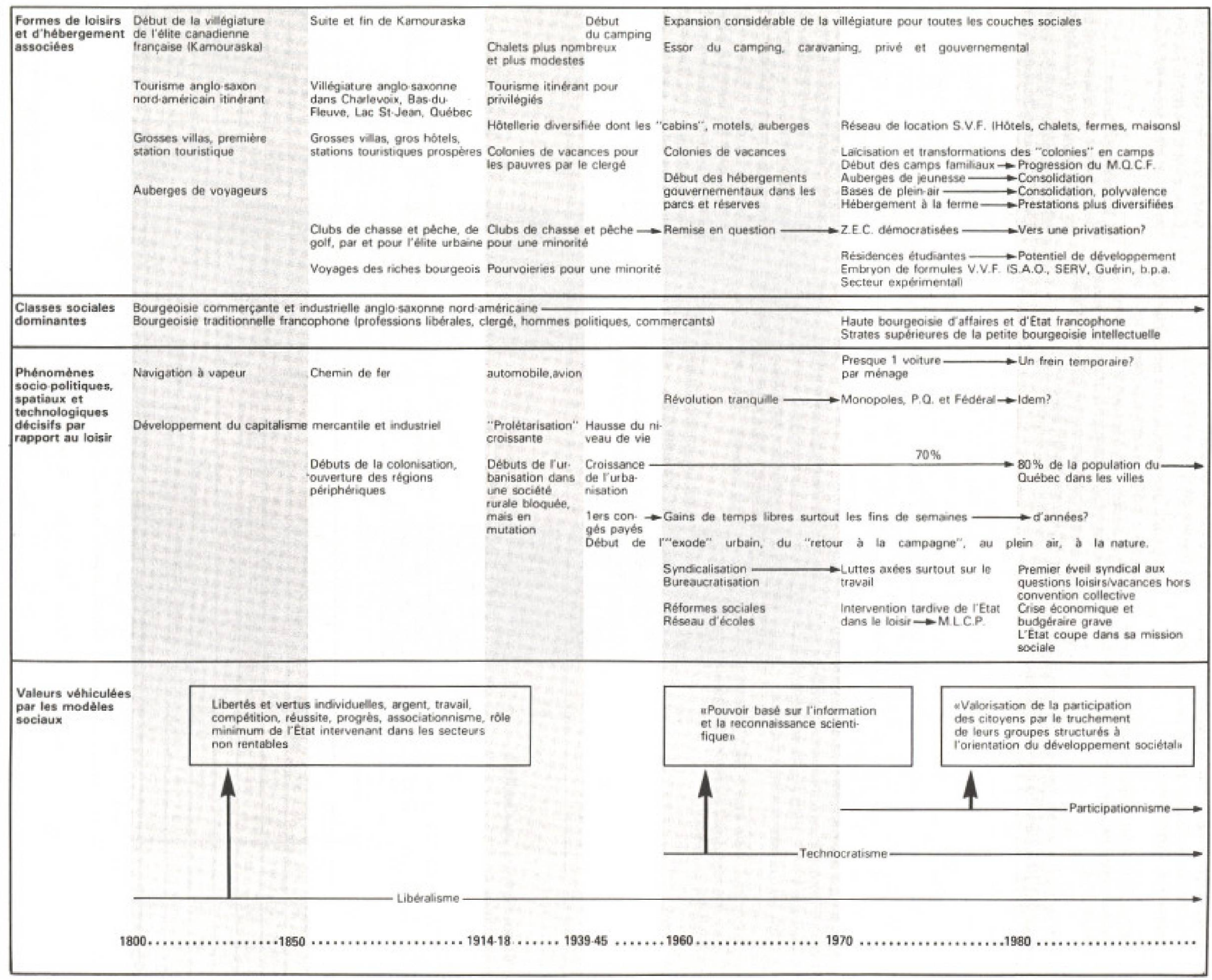


Fait par ailleurs incontestable, l'idée de consolidation et d'adaptation - recyclage des équipements actuels pour fins d'hébergement familial, émerge avec clarté. Pourquoi demandent les praticiens-acteurs des formuless actuelles, risquer une coupure du MLCP qui les alimente depuis une décade et plus, au profit d'une formule importée, extrêmement coúteuse du moins le village classique - et qui ne garantit évidemment pas aux subventionnés actuels des lendemains qui chantent? A cet égard, l'expérience francaise de la transition maison familiale $N . V . F$, est lourde d'enseignements qui confirment cette appréhension.
Si l'on élargit le débat à l'échelle de tout le patrimoine immobilier socio-touristique analysé plus haut, une solution claire se dégage parmi d'autres: l'utilisation de plus en plus rationnelle des hébergements disponibles, à faible taux d'occupation annuelle: chalets, auberges et hôtels, résidences chez l'habitant, fermes, résidences étudiantes, etc... Les chalets privés en particulier, par l'importance absolue et relative de leur nombre au Québec, devraient en toute logique fournir un pourcentage plus élevé de locations, même si la tradition répu. gne à cette pratique pour $90 \%$ des propriétaires.
Le village éclaté apparait fournir une base organisationnelle intéressante pour démar. rer ce processus, non seulement auprès de 167000 villégiateurs du Québec, bien sür, mais aussi des milliers d'autres intervenants éventuellement susceptibles d'adhérer à une pareille formule sur des territoires municipaux a bon potentiel. Conditions nécessaires: volonté politique du MLCP et sommes correspondantes pour soutenir financière. ment des études du GRTS et éventuelle. ment le minimum d'infrastructures et d'animation nécessaires à la viabilité d'un, deux ou trois projets, bien identifies, d'ici cinq ans. Un rêve... à matérialiser!

\section{Notes}

(11) Gette caricstue a it delaut de insquer des totnements

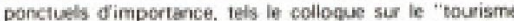

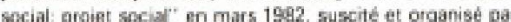

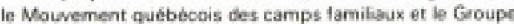
ressources wacances sites IG.A.S.

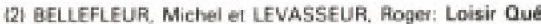

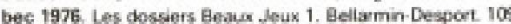

13i Sur is base des regoions administratives

(4) CLUZEAU, Parrick en MEUNIER, Gilles. Une analyse du reseau d' duablissement d'accueil et d'hébergement du Qudbec utilisables pour des vaeanées tamiliales. Doci.

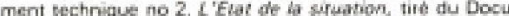

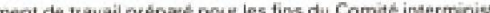
lel ser hes villo Oucbec, juiles 1980, 123 pages

6) Cetie unite se tradut partos en nombre de lits at nombe He places si a la limite, les ecars numeriques enre ces trois

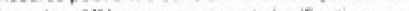
fique, les diffetenees sont peut Mgnificalives pour ces thpe: di deablissements. Cos le "tourist day" ou is "jounde de sejour": unite statistique qui conespond à un visiteur pas sant un pur dans un endroit touristique donnet in: Vocabu. latre du tourisme anglais français. Ch. Dupont, Lingui wech, Miti. 1979. D. 92 66i Quebec, MTCP, bilan satistique 1977.197879, duébec

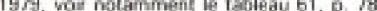

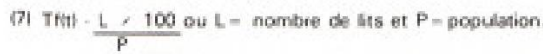
Voin a ce sujet Asperts teconomiquets du tourisme. A BAFETJE et P. DEFERT, Coll. L'Adminisiration nosuelle, Ed Buger-Leurault. Pais, 1972, ch. 2

401 Nouvesu nogionyme suggerd pour le Saguenay Lac Si Jean 49! BELLEFLUA, Mchel et LEVASSEUA, Pager. Loisir quebec 1976. Coll. Les Dosser's Beauk. Jeuk Bellarmin Desson, 1976

110 loid p. 69

111) toid a. 38

112 Defintan de Gerald FOATIN in Participstion et socesed dan Economies et Socidtes, tome $V$, no 19 , sept 70 , p. 1600 es suiv.

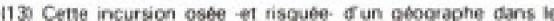

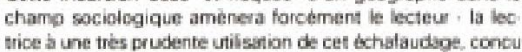

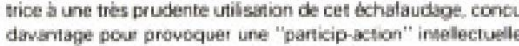
qu'une béate contemplation de l'cesure.
(14) Donnéas tireens de:

Les grands trais de rivolition of poursme ou Outhuc, Pager GRIERE, in Bullatin de I'Association des gAtogra phes de I'Amérique francaise, no 11, septembre 196. P. as a 55

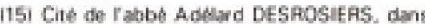

"Is colonie des Gréves" in Loisir Plus, sept. 1977, no. B1, 1. 22

116i Draneảs R. WOLFE, cité đans La vilidgiature au Qubbec. J.Piene ST-AMOUR, Fditans Asticou, Hul, 1979. o. 20

117 Alain TOURAINE La société post-industrialle. Denaet Paris. 1906.

1181 Denis POULET, Le camping en ISOO, Loigirs et Sports, juin

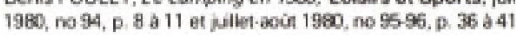

n9y Bilan de th consultation concernant les posssibiliths a conditions de développement de ta formule (VVF) Villages. Vacances. Familles au Quebec. Governement du Quebec, MLCP, Quebec, jaillet 1982, 81 peges.

Col Goivernement du Quabec. On a un mande a recrebr, Livi Blane sut be bise au Queber. 1979. Le ministre Claude Chas ron. 1979 , p. 58

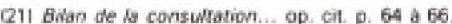

Q21 La formule Vallage Vacances.Familles RV V.F.li au Qut bec. aput 1980; p. $\mathrm{se}$

\section{Bibliographie}

BARETJE, Ri., et DEFERT, P. Aspects économiques du tourisme. Coll. L'Administration nouvelle. Ed. Berger-Levrault. Paris, 1972, ch. 2.

BELLEFLEUR, Michel, LEVASSEUR, Roger, "Loisirs Québec 1976", Les Dossiers Beaux-Jeux 1. Bellarmin-Desport, 1976, 109 pages.

BRIERE, Roger. Les grands traits de /'evoJution du tourisme au Québec in Bulletin de I'Association des géographes de I'Amérique francaise, no 11, sept. 1967. p. 83 à 95 .

DESY, Jean. Présentation de la situation du tourisme social au Québec. in Tourisme social: Besoin nouveau, réponse moderne. Rapport du congrès 1980, BITS, 24 au 31 aoùt 1980. Québec, Montréal, p. 221 à 249

GROUPES RESSOURCES- VACANCES SITES, Les belles histoires des camps familiaux. Informons-nous, special, no 2, mai 1979, 31 pages.

GRVS. Les vacances-familiales et communautaires. Situation et perspectives 80. par Richard Nicol, Congrès 1979 de I'ATT, Le Tourisme horizons 1980, Québec, 28,29 et 30 mars 1979.12 pages, reprog.
INRS-CRUR. Régions sud: le tourisme, Rapport terminal du module Tourisme, Projet Sud, mars 1972. Montréal, 159 pages et bibliographie.

Ivoir en particulier le ch. 10 de la 3ième partie: Le tourisme social au Québec, p. 119 à 136.1

POULET, Denis. Le camping en 1990 , in Loisirs et sports, juin 1980, no 94, p. 8 à 11 et juillet-aoút 80 , no $95-96$, p. 36 à 41 .

QUEBEC. On a un monde à recréer. Livre blanc sur le loisir au Québec. Claude Charron, ministre. Editeur officiel du Québec. 1979,107 pages.

QUEBEC-MLCP. Une anlyse du réseau d'établissements d'accueil et d'héberge. ment du Québec, utilisables pour des vacances familiales. Dossier technique no 1. L'État de la situation, par Patrick Cluzeau et Gilles Meunier. Québec. Juillet 1980, 123 pages.

QUEBEC-MLCP. La formule VillagesVacances-Familles au Québec. Possibilités et conditions de développement. Rapport du comité conjoint OPDQ-MLCP sur les Villages-Vacances-Familles. Québec. aoūt 1980, 134 pages.
QUEBEC-MLCP. Bilan de la consultation concernant les possibilités et conditions de développement de la formule (VVF) Villages-Vacances-Familles au Québec. Québec, juillet 1982, 81 pages.

QUEBEC-MLCP. Bilan statistique 1977-78-79. Gouv, du Québec, 1979

QUEBEC-OPDO. Dossier d'analyse et d'inventaire. Les caractéristiques secto. rielles interrégionales. Cahier IV. Les équipements et les services. 1976.

RONLO-Table sectorielle du Tourisme. Séminaire sur I'implantation des villages-vacances-familles au Québec Compte rendu synthese. Tenu les 29,30 avril et 1er mai 1981 à l'Institut Coop. Desjardins, Lévis, 92 pages.

RONLQ. Des villages-vacances-familles au Québec: de l'importation française ou une "re-création" québécoise? par Daniel Rocque et André Sénécal, Montréal, avril 1981, 62 pages (consulter en particulier les ch. 2 et 3 de la partie 21 .

ST-AMOUR, Jean-Pierre. La villégiature au Québec. Problématique de l'aménage ment du territoire. Editions Asticou, Hull, 1979, 178 pages.

TOURAINE, Alain, La Société post industrielle. Denoël, Paris, 1969. 